\title{
Magnetospheric Multiscale Instrument Suite Operations and Data System
}

\author{
D.N. Baker ${ }^{1}$ - L. Riesberg ${ }^{1}$ • C.K. Pankratz ${ }^{1}$. \\ R.S. Panneton ${ }^{1}$ - B.L. Giles ${ }^{2}$ F.D. Wilder ${ }^{1}$ - R.E. Ergun ${ }^{1}$
}

Received: 12 November 2014 / Accepted: 22 December 2014 / Published online: 4 February 2015

(C) The Author(s) 2015. This article is published with open access at Springerlink.com

\begin{abstract}
The four Magnetospheric Multiscale (MMS) spacecraft will collect a combined volume of $\sim 100$ gigabits per day of particle and field data. On average, only 4 gigabits of that volume can be transmitted to the ground. To maximize the scientific value of each transmitted data segment, MMS has developed the Science Operations Center (SOC) to manage science operations, instrument operations, and selection, downlink, distribution, and archiving of MMS science data sets. The SOC is managed by the Laboratory for Atmospheric and Space Physics (LASP) in Boulder, Colorado and serves as the primary point of contact for community participation in the mission. MMS instrument teams conduct their operations through the SOC, and utilize the SOC's Science Data Center (SDC) for data management and distribution. The SOC provides a single mission data archive for the housekeeping and science data, calibration data, ephemerides, attitude and other ancillary data needed to support the scientific use and interpretation. All levels of data products will reside at and be publicly disseminated from the SDC. Documentation and metadata describing data products, algorithms, instrument calibrations, validation, and data quality will be provided. Arguably, the most important innovation developed by the SOC is the MMS burst data management and selection system. With nested automation and "Scientist-in-the-Loop" (SITL) processes, these systems are designed to maximize the value of the burst data by prioritizing the data segments selected for transmission to the ground. This paper describes the MMS science operations approach, processes and data systems, including the burst system and the SITL concept.
\end{abstract}

Keywords Data Management · Acquisition · Science Operations · Instrument Operations · Data Products · Data Access · Data Center

$\triangle$ D.N. Baker

daniel.baker@lasp.colorado.edu

1 Laboratory for Atmospheric and Space Physics, University of Colorado, Boulder, CO 80303, USA

2 NASA Goddard Space Flight Center, Greenbelt, MD 20771, USA 


\section{Introduction}

As described in the companion chapters in this volume, the Magnetospheric Multiscale (MMS) mission consists of a highly sophisticated set of particle and fields instruments onboard four identical spacecraft flying in formation (Burch et al. 2014, this issue), together referred to as the Solving Magnetospheric Acceleration, Reconnection, and Turbulence (SMART) instrument suite.

The scientific objective of the MMS mission is to understand the microphysics of magnetic reconnection by determining the kinetic processes occurring in the electron diffusion region that are responsible for collisionless magnetic reconnection, especially how reconnection is initiated.

Specifically, and in priority order, MMS will address three specific objectives:

1. Determine the role played by electron inertial effects and turbulent dissipation in driving magnetic reconnection in the electron diffusion region.

2. Determine the rate of magnetic reconnection and the parameters that control it.

3. Determine the role played by ion inertial effects in the physics of magnetic reconnection.

The key challenge for the MMS science operations team and the individuals involved in the ground segment of the MMS mission is how to sort through the high data volumes from the $\sim 100$ individual detectors on the combined four spacecraft to identify the times when magnetic reconnection regions are being sampled. It is readily acknowledged that all MMS data will be useful for space physics understanding. But downlink priority will be given to segments when the four spacecraft are in their high-rate data collection modes precisely when sites of magnetic reconnection are being probed. Of course, this cannot be known in advance and data indicators must be carefully examined after lower resolution measurements have been collected to determine which parts of the vast onboard burst data set should properly be telemetered to the ground.

The MMS Science Operations Center (SOC) provides Phase E science leadership for this multi-institution ground segment that consists of several highly experienced teams. This ground system provides scientific leadership, operations planning, supports the on-orbit operations of the MMS observatories, supports the health and safety of the observatories, as well as providing for the production, storage, management, and dissemination of MMS science data products.

Figure 1 illustrates the role of the SOC in coordinating the Phase E science efforts between the key components of the MMS ground system.

The SOC's role in coordinating Phase E science operations among the functional elements that comprise the MMS ground system is as follows:

- Mission Operations Center (MOC). Located at the NASA Goddard Space Flight Center (GSFC) in Greenbelt, MD. The MOC is responsible for spacecraft operations, telemetry capture, and transmitting telemetry data and other operational products to the SOC. The SOC is responsible for coordinating instrument operations and providing the MOC with instrument and payload-level commanding products.

- Flight Dynamics Operations Area (FDOA). Located at the NASA Goddard Space Flight Center (GSFC) in Greenbelt, MD. The FDOA is responsible for orbit and attitude determination and control, and producing associated data for distribution to the MOC and $\mathrm{SOC}$. The SOC is responsible for dissemination of ephemeris and attitude data products to enable full understanding of the MMS science data. 


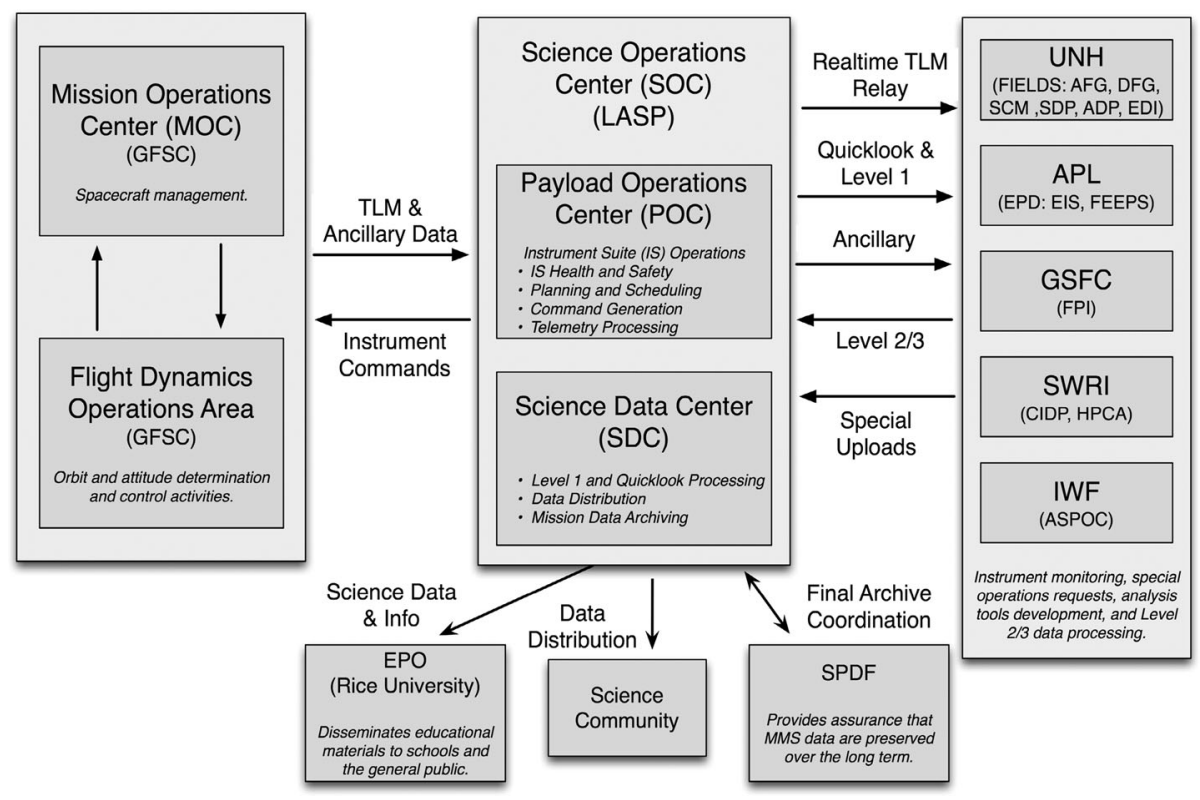

Fig. 1 Overview of the MMS ground system showing the key elements and linkages between them. Major functions for each element are also shown where appropriate

- Science Operations Center (SOC). Located at the Laboratory for Atmospheric and Space Physics (LASP) at the University of Colorado in Boulder, CO. The SOC is responsible for science operations planning, instrument Suite operations, some levels of instrument data processing, and data dissemination and archiving. The SOC consists of the Payload Operations Center (POC), responsible for instrument operations and burst data management, and the Science Data Center (SDC), responsible for science data handling and dissemination.

- Instrument Team Facilities (ITFs). Located at various institutions, described in other chapters in this volume. Instrument teams are responsible for instrument operations, health and safety, data analysis and validation; instrument monitoring and special operations requests; software for producing QuickLook and Level-2 data products; Level-2 and higher data processing. The SOC provides the operational interface between the ITFs and the MOC, processes instrument Quicklook data, and provides the official data dissemination mechanism for all levels of instrument data products.

- Education and Public Outreach (EPO). MMS education initiatives are managed by Rice University in Houston TX; MMS strategic communication activities are managed by the NASA Goddard Space Flight Center (GSFC) in Greenbelt, MD. These teams are responsible for the planning, development, review, and dissemination of educational and communication materials. The SOC supports these efforts by supplying ready access to MMS data products and providing a historical reference archive of the education and communication materials.

- Space Physics Data Facility (SPDF). Located at the NASA Goddard Space Flight Center. The SPDF serves as the long-term archive for MMS mission science data and provides a second access path to data products during the MMS mission. The SOC is responsible 
for providing the SPDF with the fully calibrated, documented data files that represent the final data products for this mission.

- Science Community. Magnetic reconnection is a fundamental physical processes of paramount importance throughout the plasma universe. The data from the MMS mission is therefore expected to be of interest to space physicists investigating a wide range of scientific problems that far exceed the priority objectives of the MMS mission itself. The MMS project, through the MMS SOC, is committed to providing ready remote access to the MMS data products and the team's analysis tools with the intent of maximizing the ultimate scientific impact of this mission.

The present chapter is devoted to describing the science operations planning processes, instrument operations management, and the definition, capture, management, and utilization of MMS data. It describes how the data flow from space to the ultimate analysis and interpretation. Section 2 summarizes, in one aggregated form, each of the MMS investigations and the types of data collected from each. This is intended to be a brief synopsis of the more extensive descriptions provided elsewhere in this volume. Section 3 describes the extensive ground segment and readers may wish to examine how the data flow from the Mission Operations facilities through to the data portals provided in the LASP science data center. As noted above, the most challenging aspect of the MMS program is to be able to select the "golden bits" of data that deal explicitly and most directly with magnetic reconnection sites on the Earth's dayside magnetopause or within the nightside magnetotail region. In Sect. 4 of this chapter, we describe how this task is undertaken. Finally, in Sects. 5 and 7 there are brief descriptions of how the MMS science teams and members on the community can access the various data products and how the data will be archived, with Sect. 6 providing a high-level overview of the MMS operations on-orbit operations scenarios-the so-called Orbit-in-the-Life. The paper concludes with a statement of data use policies and "rules of the road" for the MMS program (Sect. 8). Section 9 makes summary statements about the goals and aspirations for this remarkable flagship heliophysics mission.

\section{MMS Data Sets}

\subsection{Summary of MMS Science Instrumentation}

While the details of the MMS SMART suite of instruments are fully described in the companion chapters in this volume, the following information briefly summarizes the instruments and their measurements to support the data product descriptions that follow in this chapter. The Instrument Suite will make the required high-time resolution measurements of plasmas, electric fields and magnetic fields and provide important supporting measurements of energetic particles and ion composition. The SMART IS consists of the following complement of instruments:

- The FIELDS investigation includes a sensor suite consisting of two axial and four spinplane double-probe electric-field sensors (ADP and SDP), two flux-gate magnetometers (AFG and DFG), a search-coil magnetometer (SCM), and two electron drift instrument (EDI) per MMS spacecraft. These instruments measure the DC magnetic field with a resolution of $10 \mathrm{~ms}$, the DC electric field with a resolution of $1 \mathrm{~ms}$, electric plasma waves to $100 \mathrm{kHz}$, and magnetic plasma waves to $6 \mathrm{kHz}$ (Torbert et al. 2014, this issue).

- The Energetic Particle Detector (EPD) includes an Energetic Ion Spectrometer (EIS) and two all-sky particle samplers called the Fly's Eye Energetic Particle Sensor (FEEPS) per 


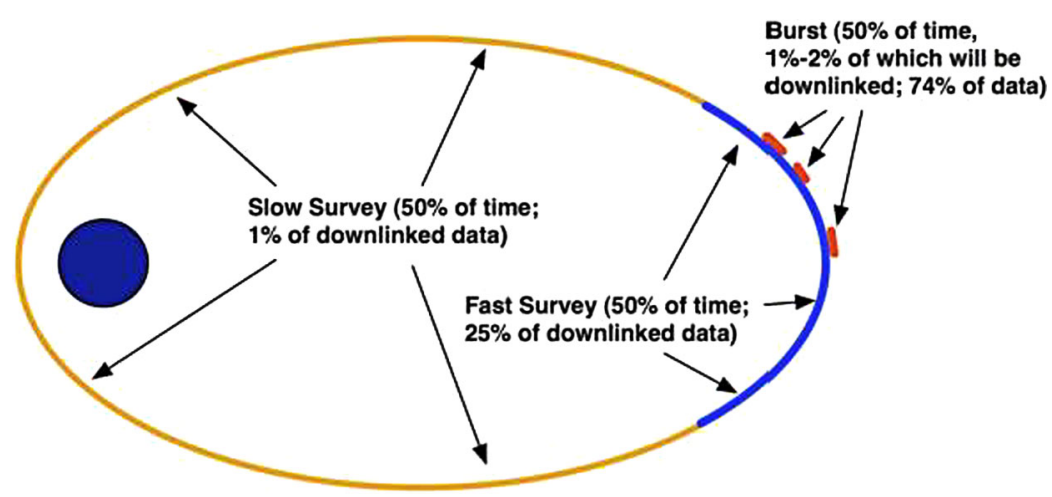

Fig. 2 Data Collection Strategy Overview. The slow survey collection period encompasses about half of each orbit period (yellow curve), but comprises only about $1 \%$ of the downlinked data. The fast survey data are collected within each orbit's region of interest for distances $>9 R_{E}$ on the day side and for distances $>15 \mathrm{R}_{\mathrm{E}}$ on the night side (blue curve) and comprise about $25 \%$ of the downlinked data. Burst intervals (red segments) will comprise $74 \%$ of downlinked data

MMS spacecraft. These instruments measure the energy-angle distribution and composition of ions ( 20 to $500 \mathrm{keV}$ ) at a time resolution of $<30$ seconds, the energy-angle distribution of total ions (45-500 $\mathrm{keV}$ ) at a time resolution of $<10$ seconds, and the coarse and fine energy-angle distribution of energetic electrons $(25-500 \mathrm{keV})$ at time resolutions of $<0.5$ and $<10$ seconds, respectively (Mauk et al. 2014, this issue).

- The Fast Plasma Investigation (FPI) includes four dual electron spectrometers (DES) and four dual ion spectrometers (DIS) per spacecraft. When the data from the two sets of four dual-spectrometers are combined, FPI is able to provide the velocity-space distribution of electrons from $10 \mathrm{eV}$ to $30 \mathrm{keV}$ and ions from $10 \mathrm{eV}$ to $30 \mathrm{keV}$ with a time resolution of $30 \mathrm{~ms}$, and $150 \mathrm{~ms}$, respectively (Pollock et al. 2014, this issue).

- The Hot Plasma Composition Analyzer (HPCA) measures the composition-resolved velocity-space distribution of ions from $1 \mathrm{eV}$ to $40 \mathrm{keV}$ with time resolution of 10-15 seconds (Young et al. 2014, this issue). There is one HPCA per MMS spacecraft.

- The Active Spacecraft Potential Control (ASPOC) generates beams of indium ions to limit positive spacecraft potentials within $+4 \mathrm{~V}$ in order to improve the measurements obtained by FPI, HPCA, ADP, and SDP (Torkar et al. 2014, this issue). There are two ASPOC per MMS spacecraft.

The instrument suite on each spacecraft is supported by a Central Instrument Data Processor (CIDP), which collects and stores telemetry from each instrument and sends it to the spacecraft for downlink. The CIDP provides time and spin synchronization as well as switched power services to the instruments, and forwards commands received from the spacecraft flight computer.

\subsection{Overview of Science Data Types and Collection Strategy}

The most significant challenge to MMS Science Operations Management is the considerable disconnect between the measurement capabilities of the instrumentation and the spacecraft downlink data rate. As illustrated in Fig. 2, while MMS has two basic instrument science observing modes-slow survey and fast survey - there are three data acquisition ratesslow survey, fast survey and burst. Available data products are summarized in subsequent sections, and fall into the following three types: 
Survey data: These are low-to-medium resolution data that are collected and stored continuously. The intent is for all survey data to be telemetered to the ground each orbit. Survey data are further categorized as Fast Survey or Slow Survey data. Fast survey data have resolutions comparable with previous magnetospheric missions. Slow Survey data sampling rates are minimized to reserve data storage space for the regions of interest.

Burst data: These are the highest resolution data that are stored when instruments are operating in their Fast Survey (FS) Mode. Burst data are not stored during Slow Survey (SS) Mode. Burst data are stored in the MMS mass memory system and only the most scientifically interesting segments are selected for transmission to the ground.

Trigger data are generated by the instruments continuously when in Fast Survey Mode. These data are used by the CIDP onboard and/or by the SOC ground system element to determine the scientific relevance of the burst data segments collected, enabling the selection of specific burst data segments for transmission to the ground. A set of trigger data is generated once every $\sim 10$ seconds within the regions of interest.

Outside the primary observing region of interest, slow survey mode acquires data at a time resolution that is relatively low to conserve data storage space for the region of interest, approximately half of the orbit period. Within the region of interest, the instruments are operated in fast survey mode and data are acquired at both fast survey and burst rates. All data acquired at both slow and fast survey rates are stored onboard and transmitted to the ground. All data acquired at burst rate are stored onboard. Burst data triggers are calculated, transmitted, and used, along with the survey data when possible, to determine which burst data to transmit to the ground. On a daily basis, approximately $75 \%$ of the telemetry bandwidth will be allocated to burst data downlink, although it must be noted that this only permits transmission of $\sim 1-2 \%$ of the burst data stored on the observatories.

\subsection{Data Level Descriptions}

The MMS project will produce, manage, and disseminate multiple categories of data, each of which has a specifically defined level of refinement. MMS follows the data level definitions indicated in Table 1.

\subsubsection{Level-1 Data}

Level-1 data products are fully decommutated but constitute uncalibrated raw data at full resolution that are time-referenced. Generally speaking, Level-1 data products do not provide scientific value to the science community, as they are uncalibrated or partially calibrated only. As such, these data products will not be provided via publicly accessible interfaces.

\subsubsection{QuickLook Data}

QuickLook data are scientific data products that are rapidly generated using simplified science processing algorithms and/or with provisional calibrations. These data are intended to provide basic scientific insight, but are generally not usable for scientific studies. Generation of these data will occur within hours of receipt, whereas routine production of Level-2 and Level-3 products will generally take considerably longer.

The MMS Science Data Center (SDC) produces QuickLook science data products, which may be browsed via a website interface hosted by the MMS SOC. QuickLook data are made available to the science community within 24 hours of telemetry receipt at the SOC. QuickLook data products are described in Table 2. 
Table 1 MMS-SMART Data Level Definitions. Each instrument team bases their data products on this set of guiding definitions

\begin{tabular}{ll}
\hline Data Level & Brief Description \\
\hline Raw & $\begin{array}{l}\text { Raw telemetry data as received at the ground receiving station or ground test Ground } \\
\text { Support Equipment (GSE), organized by contacts or ground test. Data sets may over } \\
\text { and/or contain communication artifacts }\end{array}$ \\
Level-0 & $\begin{array}{l}\text { Reconstructed, unprocessed instrument, payload, and spacecraft data; any and all } \\
\text { communications artifacts, e.g. synchronization frames, communications headers, } \\
\text { duplicate data are removed. These data will typically consist of binary Consultative } \\
\text { Committee for Space Data Systems (CCSDS) packets }\end{array}$
\end{tabular}

Level-1A Fully decommutated but uncalibrated raw data at full resolution; time-referenced; "extracted telemetry items"

Level-1B Level-1A (extracted telemetry items) to which engineering (e.g. simple polynomial) calibrations have been applied; data have been annotated with ancillary information (e.g., ephemeris, attitude data) and initial instrument-level science calibrations have been applied

QuickLook Scientific data products that are generated using simplified science processing algorithms and/or with provisional calibrations. These data are intended to provide basic scientific insight. Generation of these data will occur as quickly as possible, whereas routine production of Level-2 and Level-3 products may take considerably longer. In many cases, Level-1B products will serve as QuickLook data

Level-2 Level-1 data that have been processed to physical units and/or derived geophysical parameters by combining calibration, ancillary, and other data. These data represent the lowest level of research grade scientific data, and exist at the same time and/or spatial resolution as Level-1 data

Level-3 Mission Level Data Products (MLDP). These data have been resampled spatially and/or temporally and may have been combined with measurements from other MMS instruments to produce a merged data set

Table 2 MMS-SMART QuickLook data products

Investigation Description

Fast Plasma Investigation (FPI)

Hot Plasma Composition Analyzer (HPCA)

FIELDS

Energetic Particle Detector System (EPD)

Active Spacecraft Potential Control (ASPOC)
Energy-azimuth-zenith angle vs. time spectrograms (survey "sky-maps"), and survey moment time-series including densities, velocities, and temperatures. Unpacked and de-spun FPI Trigger Terms (electron/ion "pseudo-moments")

Fluxes and moments in spacecraft coordinates (near GSE)

DC electric field vector, DC magnetic field vector, spacecraft potential, E and B wave spectra, EDI ambient electron counts when available

Electron Omni-directional spectra versus time and position. Proton Omni-directional spectra versus time and position. Pitch Angle versus Intensity for selected species and energies as a function of time and position. Solar angle versus Intensity for selected species and energies as a function of time and position. Angle-angle (All-Sky) distributions for selected species and energies and at a specified time cadence. Composition Index

24 hour plot of the ion beam current and status of the instrument for each of the two ASPOC units 


\subsubsection{Level-2 Data}

Level-2 data are derived from Level-1 data that have been processed to provide physical units and/or derived geophysical parameters by combining calibration, ancillary, and other data. These data represent the lowest level of research grade scientific data, and exist at the same time and/or spatial resolution as Level-1 data. All data products will include data quality indicators and uncertainties as appropriate to aid in correct use.

Production of Level-2 science data products and delivery to the SDC is the responsibility of each instrument team. For convenience, some Level-2 data products are generated at the SDC using software maintained by instrument team personnel. Level-2 data products are summarized below in Tables 3, 4, 5, 6 and 7. A detailed description of all MMS Level2 data products, including file formats and naming conventions, is provided in the MMS Instrument Suite Science Data Products Guide, which will be available from the SDC postlaunch.

Table 3 Fast Plasma Investigation (FPI) Level-2 science products for the electron (DES) and ion (DIS) sensors

\begin{tabular}{|c|c|}
\hline Sensor(s) & Description \\
\hline \multirow[t]{3}{*}{ DES/DIS } & $\begin{array}{l}\text { Energy, azimuth, zenith-angle spectrogram ("sky/burst-map") time-series supporting various } \\
\text { levels of time axis zoom for slow survey, fast survey, and burst records }\end{array}$ \\
\hline & $\begin{array}{l}\text { Velocity distributions (with spacecraft potential corrections) in 3D: supporting various slices, } \\
\text { projections, or reductions (pitch angle, ecliptic, GSM equator, etc. portrayals) }\end{array}$ \\
\hline & $\begin{array}{l}\text { Moments (density, velocity vector, temperature vector, pressure tensor, heat flux vector) for } \\
\text { survey and bursts }\end{array}$ \\
\hline
\end{tabular}

Table 4 Hot Plasma Composition Analyzer (HPCA) Level-2 science products

\begin{tabular}{ll}
\hline Sensors & Description \\
\hline HPCA & Calibrated \& background-corrected energy flux for $\mathrm{H}^{+}, \mathrm{He}^{+}, \mathrm{He}^{++}$, and $\mathrm{O}^{+}$ions \\
& Velocity distribution functions of $\mathrm{H}^{+}, \mathrm{He}^{+}, \mathrm{He}^{++}$, and $\mathrm{O}^{+}$ions derived from j(E) \\
& Moments (density, velocity, temperature) \\
\hline
\end{tabular}

Table 5 FIELDS Level-2 science data products

\begin{tabular}{ll}
\hline Sensor(s) & Description \\
\hline AFG & 3-component B-field to 128 vectors/sec \\
DFG & 3-component B-field to 128 vectors/sec \\
SDP-ADP & 1 spacecraft potential sample \\
SDP-ADP & 3-component DC E-field to 8192 samples/sec \\
SDP-ADP & 3-component AC E-field to 8192 samples/sec \\
SDP-ADP & 3 sphere voltages from ADP and SDP \\
SDP-ADP & 3-component high speed burst E-field, to \\
& 65536 samples/sec \\
SDP-ADP & 3-component Low Frequency (LF) Electric \\
& Spectra, 1-8000 Hz \\
SDP-ADP & 3 sampled Medium Frequency (MF) Electric \\
& Spectra, .25-100 kHz \\
\hline
\end{tabular}


Table 5 (Continued)

\begin{tabular}{ll}
\hline Sensor(s) & Description \\
\hline SCM & $\begin{array}{l}\text { 3-component Low Frequency (LF) Magnetic } \\
\text { Spectra, .2-6000 Hz } \\
\text { 3-component high speed AC B-field } \\
\text { waveform, to 16384 samples/sec }\end{array}$ \\
& 3-component AC (alternating current) B-field \\
SCM & Electric fields and drift velocity \\
EDI & Ambient electrons at two directions \\
EDI & 3-component E-field, to 8192 samples/sec \\
SDP-ADP-EDI & 3-component combined B-field from all Mag \\
AFG-DFG-SCM & sensors, to 1024 or greater vectors/sec \\
\hline
\end{tabular}

Table 6 Energetic Particle Detection (EPD) system Level-2 science products

Sensor(s) Description

FEEPS

FEEPS/EIS

FEEPS

FEEPS/EIS

FEEPS

EIS

EIS

EIS

EIS
L2-FEEPS-Electron-Burst: Calibrated (Intensity) electron data cubes: Intensity $\times$ Energy $\times$ Detector versus time, sampled at the instrument sampling rate combined for both heads (18 detectors total) with each detector mapped to a Geophysical-Coordinate look direction and magnetic field direction (64 spin sectors)

L2-Fast-Ion-Burst: Calibrated (Intensity) Fast Ion data cube: Intensity $\times$ Energy $\times$ Detector versus time, at the slower of the 2 instrument sampling rates, combined 2 FEEPS heads ( 6 detectors) and the 1 EIS head ( 6 detectors) with each detector mapped to a Geophysical-Coordinate look direction and magnetic field direction (32 spin sectors)

L2-FEEPS-Electron-Survey: Calibrated (Intensity) electron data cubes: Intensity $\times$ Energy $\times$ Sector Elevation $\times$ Sector Longitude versus time, sampled 1/8 spin period combined for both heads with each sector mapped to a Geophysical-Coordinate look direction and magnetic field direction

L2-Fast-Ion-Survey: Calibrated (Intensity) Fast Ion data cube: Intensity $\times$ Energy $\times$ Sector Elevation $\times$ Sector Longitude versus time, at 1/8 spin period timing, combined 2 FEEPS heads ( 6 detectors) and the 1 EIS head ( 6 detectors) with each sector mapped to a Geophysical-Coordinate look direction and magnetic field direction

L2-FEEPS-Integral-Electron-Burst: "all-sky" sampling of the electron intensities during Burst periods at a $12 \mathrm{msec}$ cadence for 3 broad energy ranges.

L2-EIS-PhxTOF-Ion-Burst: Calibrated ion intensities listed as a function of mass species ( $\mathrm{H}$ and $\mathrm{O}$ ), energy, and look direction for the lower energy range (derived from time-of-flight $\times$ Pulse Height) of the EIS sensor with viewing directions mapped to magnetic field direction and geophysical coordinates. 32 samples per spin

L2-EIS-ExTOF-Ion-Burst: Calibrated ion intensities listed as a function of mass species $(\mathrm{H}, \mathrm{He}$, and $\mathrm{O})$, energy, and look direction for the higher energy range (derived from time-of-flight $\times$ Energy) of the EIS sensor with viewing directions mapped to magnetic field direction and geophysical coordinates. 32 samples per spin

L2-EIS-PhxTOF-Ion-Survey: Calibrated ion intensities listed as a function of mass species ( $\mathrm{H}$ and $\mathrm{O}$ ), energy, and look direction for the lower energy range (derived from time-of-flight $\times$ Pulse Height) of the EIS sensor with viewing directions mapped to magnetic field direction and geophysical coordinates. 8 samples per spin L2-EIS-ExTOF-Ion-Survey: Calibrated ion intensities listed as a function of mass species ( $\mathrm{H}, \mathrm{He}$, and $\mathrm{O}$ ), energy, and look direction for the higher energy range (derived from time-of-flight $\times$ Energy) of the EIS sensor with viewing directions mapped to magnetic field direction and geophysical coordinates. 8 samples per spin 
Table 7 Active Spacecraft Potential Control (ASPOC) Level-2 science products

\begin{tabular}{ll}
\hline Sensor(s) & Description \\
\hline ASPOC & $\begin{array}{l}\text { Ion beam current for each of the two ASPOCs and sum of } \\
\text { the currents from the two ASPOCs at } 1 \text { second time } \\
\text { resolution } \\
\text { Ion beam energy for each of the two ASPOCs at } 1 \text { second } \\
\text { resolution }\end{array}$ \\
\end{tabular}

\subsubsection{Level-3: Mission Level Science Products}

The MMS Mission Level Data (MLD) products are resampled spatially and/or temporally and are combined with measurements from other MMS instruments to produce a merged data set. Level-3 data may include other publicly available data products if they are of significance to the MMS science efforts and can be merged into the data system at the SDC (e.g. geomagnetic indices, solar wind parameters, etc.).

All "mission-level" (Level-3) products are provided only for designated events. Specific MMS Level-3 products, along with responsibility for their generation, are indicated in the MMS Instrument Suite Science Data Products Guide.

\subsection{Data Product File Formats}

The MMS project uses the Common Data Format (CDF) for all publicly available scientific data products. This format is intended to be familiar and convenient for the scientific communities that the MMS program serves. The utilization of a common file format facilitates the use of MMS data in multi-mission science studies and allows the data to be readily used with existing user tools. CDF is used by several other NASA missions, including Cluster, THEMIS and the Van Allen Probes, and is an actively supported product of NASA's Space Physics Data Facility (SPDF).

\subsection{Data Product Volume}

The MMS-SMART instrument suite will produce a substantial volume of data on a daily basis. Level-2 data products alone are expected to account for more than 50 GB per day for all four observatories. Table 8 indicates the expected data volumes for each MMS-SMART investigation's data products.

\section{MMS Ground Segment}

The MMS ground segment is comprised of the elements needed to operate the MMS observatories and perform data handling functions, and is illustrated in Fig. 3, below.

The Mission Operations Center (MOC) provides the necessary interfaces with the Ground System Network and the SOC to facilitate the transfer of science data and commands with each spacecraft in the constellation.

The SOC operates the MMS instruments suites in partnership with instrument teams, including routine and non-routine operations. Key SOC operations functions include routine planning and scheduling, command generation and uplink via the MOC, health and safety assessment, contingency response, onboard and ground-based data management, and dissemination of data. Onboard burst data management and science data downlink is a key 
Table 8 MMS-SMART data volume (daily, uncompressed, for all 4 spacecraft)

\begin{tabular}{lcrrrr}
\hline MMS Data Volume (daily, per spacecraft, megabytes) & & & & \\
\hline Instrument & $\begin{array}{l}\text { Raw } \\
\text { Volume }\end{array}$ & $\begin{array}{l}\text { Level-0 } \\
\text { Volume }\end{array}$ & $\begin{array}{l}\text { Level-1 } \\
\text { Volume }\end{array}$ & $\begin{array}{r}\text { Level-2 } \\
\text { Volume }\end{array}$ & Total \\
\hline Fast Plasma Investigation & 1048 & 1048 & 5240 & 44800 & 52136 \\
Hot Plasma Composition Analyzer & 236 & 236 & 1180 & 1772 & 3424 \\
Fluxgate magnetometer-AFG & 12 & 12 & 60 & 152 & 236 \\
Fluxgate magnetometer-DFG & 8 & 8 & 40 & 128 & 184 \\
Search-coil magnetometer & 140 & 140 & 700 & 1040 & 2020 \\
Spin-plane electric field instrument & 308 & 308 & 1540 & 2400 & 4556 \\
Axial double-probe electric field & 156 & 156 & 780 & 1200 & 2292 \\
instrument & 28 & 28 & 140 & 400 & 596 \\
Electron-drift electric field & & & & & 740 \\
instrument & 20 & 20 & 100 & 604 & 744 \\
Energetic Particle Detector & 5 & 5 & 32 & 32 & 72 \\
Active Spacecraft Potential Control & & 2000 & 10000 & 52500 & 66000 \\
Total (approx.) & 2000 & & &
\end{tabular}

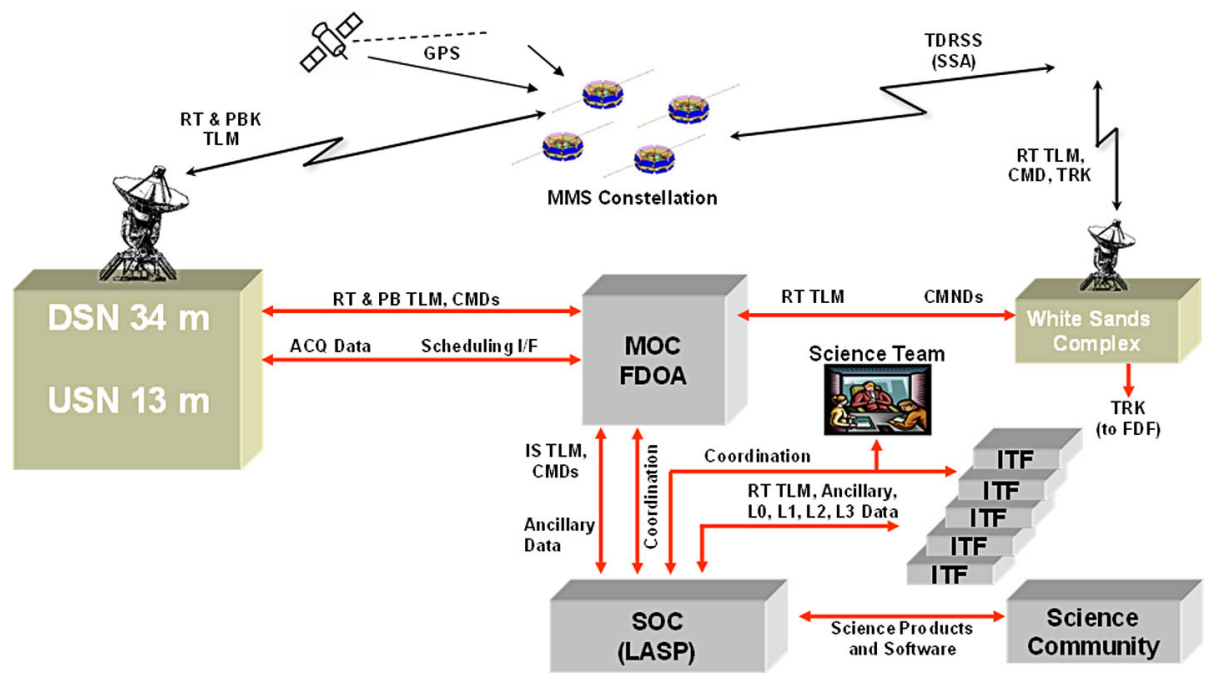

Fig. 3 MMS ground system overview showing the relationships between the key ground system elements, notably the Mission Operations Center, the Flight Dynamics Operations Area, and the Science Operations Center. Realtime (RT) telemetry (TLM) flow from the observatories through the MOC to the SOC and are relayed to instrument teams. Commands (CMDs) are formulated at the SOC and uplinked via the MOC. Tracking data (TRK) are obtained via White Sands and combined with onboard GPS data to facilitate spacecraft navigation

function, requiring ground-based assessment of onboard data and selection. To facilitate downlink of the optimal science data, the SOC computes a Figure of Merit (FOM) for onboard burst data, and selects the best subset of collected data for downlink. The SOC also 
provides a Scientist-in-the-Loop interface that enables the capability for a scientist to change the FOM, adjust the start/end times, or otherwise prioritize burst data for downlink.

The Deep Space Network (DSN) will be the primary high-bandwidth communication link used to downlink science data from the MMS observatories. The Universal Space Network (USN) will be used a backup communications link when DSN is unavailable. The Tracking and Data Relay Satellite System (TDRSS) will provide a low bandwidth communication link that can be used to telemeter some spacecraft housekeeping data and uplink some commands but cannot be used for science data downlink. The MOC provides and manages the interfaces between the ground communication networks and the SOC.

\subsection{Ground System Data Flow}

Responsibility for MMS data handling, including data processing and analysis activities, is a distributed function within the MMS mission, with each MMS instrument team having shared responsibility for generating and maintaining the MMS data during the active mission. Data capture of both instrument and spacecraft recorded telemetry occurs at the MOC. During ground station contacts, real-time telemetry are relayed from the MOC to the SOC. Following each ground station contact, the MOC provides the SOC with recorded telemetry frames containing instrument and spacecraft data, along with appropriate status and ancillary information. The SOC performs Level-0 processing on these data, dividing the telemetry data streams into packets, and making these data available for download by the MMS instrument teams. The packet sets are then processed at the SOC to form Level-1 data products, which are used to monitor state-of-health and to support operations. The Level-1 data products are also used to support Scientist-in-the-Loop decision-making and are available within 1 hour of telemetry receipt at the SOC. QuickLook science data products are created to provide a preliminary view of MMS science measurements and are accessible to the science community via the Internet within 24 hours of ground receipt.

Within one day of receipt, the SDC at LASP makes available telemetry and ancillary data to the instrument teams for Level-2 data processing. Each instrument team generates Level-2 data products based on current calibration factors and delivers these products within 30 days to the SDC for distribution and archival.

Magnetometer and ASPOC data are needed by many other instruments to generate research grade Level-2 data products, so preliminary magnetometer and ASPOC processing occurs at the SDC to facilitate shorter availability latency. In these cases, maintenance and development of the processing software, data validation, and calibrations remain the responsibility of the each instrument team, with the SDC simply executing the provided software. These data products are generated and made available to all teams within 14 days of receipt of telemetry at the SOC.

Updates to calibrations, algorithms, and/or processing software are expected to occur regularly, resulting in appropriate production system updates followed by reprocessing of science data products. Systems at the SOC and instrument team locations will be designed to handle these periodic version changes. Likewise, publicly available software tools will be updated as needed.

The SOC will maintain an active archive of all MMS data levels, and provides direct access to the MMS science team and science community through the entire lifecycle of the MMS mission. Similarly, data products are transferred regularly to the SPDF for long-term archival and complimentary science community access. 


\subsection{Inter-Element Interaction and Interfaces}

\subsubsection{MOC and POC Interaction}

In order to support operations of the MMS Instrument Suites, and in order to support capture and processing of science data, coordination and information exchanges between the MOC and the POC are necessary. The principal transfers are illustrated in Fig. 1 above, with interfaces pertaining to science-related data flows being emphasized in that figure.

All MMS telemetry data (spacecraft and instrument housekeeping and science) are captured by the MOC at Goddard Space Flight Center (GSFC) and subsequently are relayed or transferred to the POC. Immediately following a recorder dump contact with an observatory, the MOC forwards all captured spacecraft and instrument telemetry files to the POC. MMS uses the reliable file mode of the CCSDS (Consultative Committee on Space Data Systems) File Delivery Protocol (CFDP), thus minimizing needed Level-0 processing and accounting. All spacecraft, instrument, and ancillary data are transferred to the POC on an ongoing basis for archiving and distribution; however, spacecraft data are maintained in the MOC for the operational duration of the mission. The communications links used to support transfers between the MOC, SOC, and other ground system elements are private, high-reliability NASA networks. Transfers between the SOC and other ground system sites (e.g. team facilities, science community) use the Internet.

To support instrument suite operations, the MOC relays all real-time spacecraft and engineering telemetry to the POC using a private operational network. The MOC transfers all instrument recorded telemetry files directly to the POC as it is received to allow the SOC to manage and disseminate these data. In addition to telemetry data transfers to the SOC, ancillary data will be provided by the Flight Dynamics Operations Area (FDOA) to the SOC via the MOC interface. These data include predicted and definitive orbit ephemerides, spin axis attitude, spin rate and phase angle, as well as maneuver history information.

\subsubsection{POC and Instrument Team Interaction}

Successfully operating and maintaining the health of the MMS instruments suites is a joint effort involving the POC and ITF personnel. The POC coordinates with all ITFs to establish and maintain baselines of nominal operational and instrument calibration sequences for each mission phase, respective spacecraft state, and orbit event. Mission phases are introduced in Sect. 6, below and described in greater detail in Fuselier et al. (2014, this issue). Each instrument team maintains primary responsibility for their instrument operational plans and performance, with the POC integrating and executing operations plans and procedures for the entire MMS suite, including both routine and non-routine activities. For nominal operations, the POC facilitates a recurring multi-week planning cycle, in which all instrument team inputs are incorporated, and generates uplink products for handoff to the MOC for incorporation into the observatory-wide command sequence load. The POC also provides a set of web-based tools, enabling instrument teams to submit problem reports and change requests, view operational information, and to acquire the data they need to assess health and performance, and ensure the optimal operation of their instruments. Information and data provided by the POC to ITFs includes real-time telemetry data, contact schedules, planned activity summaries, and data availability notifications and quality reports. Similarly, operations support products and information are provided by ITFs to the POC, including instrument state-of-health assessments, special planning requests, and any necessary special 
uploads (e.g. flight software updates). The principal data products transferred over this interface are indicated in Fig. 1.

The POC notifies the ITFs of any anomalous conditions as they occur and participates in Anomaly Review Board (ARB) activities, as needed. The POC also notifies the ITFs of spacecraft, constellation, or ground segment configuration changes affecting previously established nominal operational command sequencing. To safely implement commanding, the POC works closely with the MOC to schedule and implement loading the ATS (Absolute Time Sequences) commands and the RTS (Relative Time Sequences) commands, which execute within the spacecraft computer (Tooley et al. 2014, this issue), as well as enabling needed real-time commanding periods.

\subsubsection{SDC and Instrument Team Interaction}

As with operations, the MMS ground data system is a shared responsibility of the SOC and ITFs. With the SDC serving as the central hub for MMS science data, the interface between the SDC and instrument teams is designed to facilitate production, management, and dissemination of science data products. Low-level telemetry data, ancillary data, and QuickLook data are generated at the SOC and made available to the teams via the SDC for generation of higher-level science products.

Within one day of acquisition, each instrument facility receives notification of the availability of Level-0 and Level-1 data. Each facility then initiates transfer from the SDC of the telemetry and ancillary data needed for Level-2 production and data validation. Each team then returns Level-2 science data for its instrument to the SDC within 30 days. As indicated in Table 1, Level-2 data will be in physical units for all appropriate quantities, based on best current calibration factors and analysis routines. Reprocessing of Level-2 data is expected to occur periodically throughout the mission as significant advances in instrument calibration and understanding warrant the production of a new data version. Version control systems are utilized by the SDC and the teams to ensure that changes in calibration and reprocessing of Level-2 data are tracked and made known to the science community via release notes and embedded metadata.

\subsection{Science Data Production}

In addition to raw telemetry and other ancillary data that are needed to support science processing by the various instrument teams, interdependencies exist between instrument processing algorithms. Data products that other teams depend on (e.g. Level 2 magnetometer data) are provided via programmer-level interfaces once those data are available at the SDC. For convenience to the teams, these interfaces work in much the same way as the interfaces that are used to access the Level-1 and ancillary data.

\subsection{Additional Data Flows}

The general science community is able to access MMS science data products (QuickLook, Level 2, and Level 3) via a publicly accessible web site and web-based data access interfaces, which are described in Sect. 5, below. 


\section{MMS Burst System}

\subsection{Overview}

At their highest resolution, the SMART instruments will produce particle and fields data at rates many times the orbit-averaged telemetry rate. These high-resolution observations, (Burst Data), are key to understanding magnetic reconnection, but are essential for only a fraction of the orbit.

Traditionally, space plasma missions have employed burst data acquisition at the instrument level (e.g., the Wind and Polar missions, Acuña et al. 1995) whereby individual instruments independently determine when to acquire burst data. The drawback of this approach is that burst data from different instruments often do not overlap. More recent missions (e.g. FAST, Ergun et al. 2001) have incorporated spacecraft-wide burst data acquisition in which a central data processing unit receives input from the instruments simultaneously.

The SMART instrument suite uses a combination of approaches. Rather than rely on triggers to turn burst data collection on or off, the SMART instrument suite will always collect burst data throughout the Region of Interest (ROI) encounters. Onboard flash memory will be used to store and manage the entirety of ROI data while ground-based software will use telemetered information to determine which onboard data buffers are most worthy to be downlinked in the limited telemetry stream. Using this approach, the most interesting concurrent data from all instruments on the four spacecraft will be downlinked for analysis, thus maximizing the science return in the expected regions of magnetic reconnection.

Given that the MMS Burst System is both crucial to carrying out the science objectives of the MMS mission, and extensive in its design, ample attention is given to this aspect of the mission in the following sections. Most of the MMS mission elements that have been mentioned previously play a key role in facilitating the burst system, and their pertinent roles are described below, as well as the mechanisms by which these key science data are acquired on the ground.

\subsection{Goals and Constraints}

The goal of the burst data system is to enable collection, on the ground, of data that have the highest probability of containing a magnetic reconnection event. To ensure a full understanding of the event, data from all four observatories covering the same time period must be received on the ground.

The volume of burst data collected on board will far exceed the downlink capabilities of the Space-to-Ground communications system (RF downlink bandwidth and Tracking Network Resources). To address this problem, the collected burst data must be evaluated prior to downlink and assigned a Figure Of Merit (FOM) so that the most promising data will be saved and subsequently brought down for processing and analysis. Onboard storage capacity for the Instrument Suite is large, but limited relative to instrument data rates. Thus, decisions on which data should be preserved must be made in a timely manner to avoid the loss of any key science data. The mechanisms employed for doing this are described below.

\subsection{Terminology and Definitions}

There are many specialized terms and approaches that have been adopted for the challenging MMS burst data system. The definitions of these terms are spelled out in Appendix. Subsequent portions of Sect. 4 describe in detail the burst system implementation. 


\subsection{Approach}

\subsubsection{Overview}

To optimize how onboard memory is utilized, and as noted previously, there are two distinct modes of data collection: Survey and Burst. The survey data stream is collected nearly continuously. When the spacecraft are not in or near targeted regions of scientific interest (about half the duration of each orbit), data are collected in the Slow Survey mode. In this mode, the instruments acquire data at significantly less than maximum data collection rates. As the spacecraft approach targeted regions of scientific interest (the remaining half of each orbit), the instruments are configured into the Fast Survey mode and acquire data at moderate time resolution. All of the data collected in survey mode are downlinked to the ground. This is depicted in Fig. 2.

The burst data stream is collected concurrently with the collection of Fast Survey data. Burst data are high time resolution, continuous data sets. As indicated previously, at this resolution the MMS instrument suite will produce scientific measurements many times faster than the orbit-averaged telemetry rate. Burst data rates are significantly higher than the downlink bandwidth available to the MMS mission, and it is not possible to downlink the entire burst data stream recorded on-orbit. Since only a small fraction of the collected burst data are essential for core science goals, a selection scheme is used to prioritize burst data for downlink.

\subsubsection{Burst Data Prioritization}

Cycle Data Quality (CDQ) Each burst buffer on each of the spacecraft spans 10 second and is assigned a Cycle Data Quality value, an integer number between 0 and 255. This number is calculated onboard by a linear combination of 34 onboard trigger data values calculated by the instruments and supplied to the CIDP:

$$
C D Q=\frac{\sum w_{i}\left(T D_{i}-O S_{i}\right)}{\sum w_{i}},
$$

where $T D_{i}$ is the trigger data value provided by the instruments, $O S_{i}$ is an offset, and $w_{i}$ is a weighting factor. These CDQ values constitute a small data volume, allowing operations personnel to downlink these data several times during an orbit.

The weighting factors and offsets are stored in tables held onboard each of the spacecraft in the CIDP. These factors and offsets are reconfigurable. Initially, the weighting factors are configured such that the CDQ increases with large changes in plasma density and reversals of the magnetic field (i.e., when the magnetopause current layer is crossed).

Mission Data Quality (MDQ) The time arrays of the CDQ values from the four spacecraft are combined to a single time array, called the "Mission Data Quality" (MDQ), by an amplitude-ordered weighting:

$$
M D Q(t)=\sum_{S C} C D Q(t)^{*} O W_{i}(t)
$$

where $O W_{i}(t)$ is the amplitude-ordered weighting and the summation is over the four spacecraft. Each element in the resulting MDQ time array represents one 10 second Trigger Cycle. Initially, $O W=[0.4,0.3,0.2,0.1]$. For each time period, the largest CDQ among the four 
spacecraft is weighted at 0.4 , the second largest is weighted at 0.3 , and so on. The time array of MDQ values reflects the data quality of the four spacecraft combined, with the heaviest weighting on the highest quality value among the spacecraft.

After the MDQ time array is calculated, the most scientifically valuable burst data are selected for downlink based on the MDQ array. An important aspect of this selection process is to have continuous stretches of data. These continuous stretches of data are called Burst Data Segments, which consist of a set of $N$ time-contiguous burst data buffers, of which each represent one Trigger Cycle. The ultimate selection of the most scientifically valuable burst data involves an optimization of MDQ values and segment lengths. Each segment is assigned a "Figure of Merit", which is an indication of its inferred scientific value and is the quantity used to prioritize data downlink.

Data Selection The burst data are selected for downlink in one of two ways. Automatic selections are made based on data quality values (see above). This system runs automatically at the SOC following each downlink of Survey data and Burst metadata. Alternatively, a scientist (the SITL) makes the selection of the burst data based on viewing the survey data and data quality values.

The Automated Burst System (ABS) utilizes the calculation of the TDNs and CDQs on board each spacecraft, as well as the MDQ and FOM values by the SOC. The goal of the ABS is to select and downlink the recorded burst data that has the highest probability of containing a magnetic reconnection event. FOM values will be used to prioritize burst segments in the downlink queues, as well as manage buffer allocations. The ABS will promote higher FOM data segments for earlier downlink while demoting the lowest FOM data segments and freeing their buffers to ensure adequate buffers are available to record new burst data. The data segments and FOM values determined by the automated algorithm from the previous orbit will be used to formulate the commands uplinked to the CIDP if no manual selections are made.

The manual selection process, known as the Scientist-in-the-Loop (SITL), is also available to help ensure that the burst data with the highest science value is selected. The lead scientist who serves in this role at any given time is known as the Designated Scientist. The Designated Scientist has at his/her disposal the survey data from MMS, data from other satellites, and ground-based data such as magnetometer chains. Additionally, a Graphical User Interface (GUI) software tool called "EVA" will be available to the Designated Scientist to assist in selecting burst data. EVA was developed by the MMS Interdisciplinary Science Team at the University of California, Berkeley, with assistance from both the SDC and scientists at the University of Colorado. The software interacts with the MMS ground system via the SDC and can display a time series of FOM values for the most recent orbits, as well as the associated survey data. The Designated Scientist may modify the time length of burst segments, as well as their FOM value, and transmit these to the SDC in order to optimize the burst data downlink. EVA also includes validation to ensure that the selections made by the Designated Scientist will not lead to unintended difficulties in managing the downlink of burst data. These validation criteria include limits to the length of a burst segment, as well as the number of intervals with unusually large FOM values. This manual selection processes can be used to validate the automatic selections and/or override the automatic process potentially allowing for a much more informative selection of burst data.

\subsubsection{Multiple Orbit Data Collection}

The burst data selection system, diagrammed in Fig. 4, also allows for onboard retention of data from previous orbits. Past space missions operating in the magnetosphere have in- 


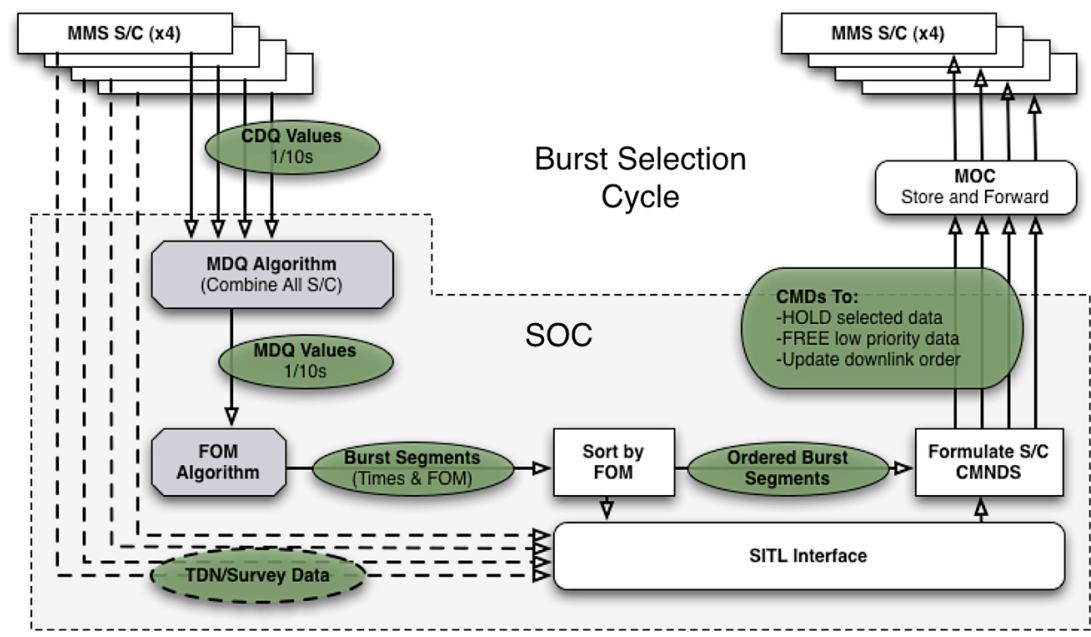

Fig. 4 Burst data selection process and SOC data flow as described in the text. Both automated and SITL-defined burst selections are possible

dicated that many reconnection events may occur in a single day, whereas other days may have few such events. While available space-to-ground data bandwidth limits the average burst data downlink to roughly 15 minutes of burst data per day, the MMS flight segment allows for storage of a large quantity of data selected by either the automatic routine or the SITL scientist. This long-term retention of selected data permits optimization of the number of reconnection events and transmission to ground of these events in a pre-determined (by FOM) priority order over the period of several orbits. In this way, lower-priority measurements can be sacrificed to enable the full downlink of those events that are most interesting from a scientific perspective.

\subsection{Burst Data System Responsibilities}

The functions necessary to implement the burst data system are distributed between flight and ground segment components as shown in Fig. 5, and are described below.

\section{Flight Segment Elements:}

- Each instrument suite contains the instruments and associated processing elements, which generate the burst data and burst metadata as well as the memory storage elements needed to store the data until it can be assessed and downlinked when selected for further analysis.

- Each MMS spacecraft provides the capabilities necessary to transmit data from the instrument suite to the ground and to receive and transfer command information from the ground to the instrument suite.

\section{Ground Segment Elements:}

- The Ground System Network provides the communication resources required to transfer data to and from each observatory in the MMS constellation.

- The MOC provides the necessary interfaces with the Ground System Network and the SOC to facilitate the transfer of science data and commands with each spacecraft in the constellation. 


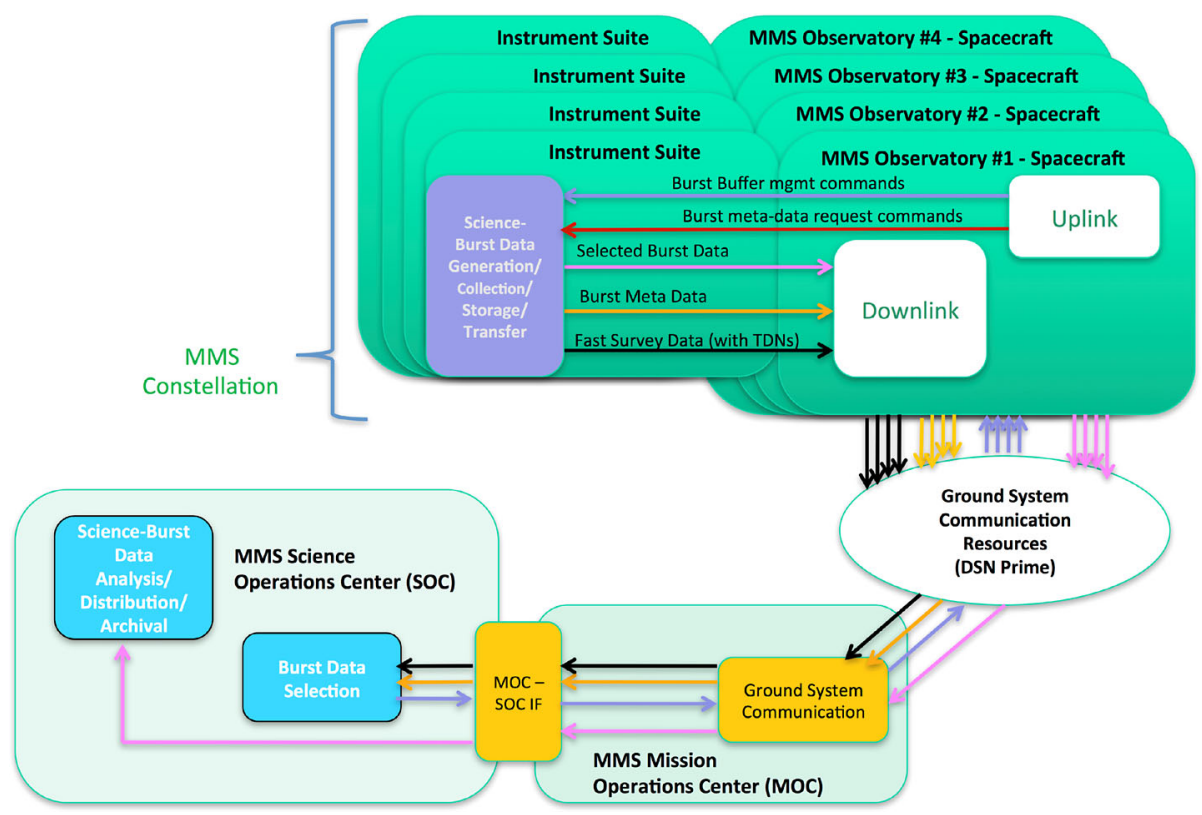

Fig. 5 The MMS Burst Data system involves key interplay between all four MMS spacecraft, the Mission Operations Center, and the Science Operations Center as described in the text

- The SOC provides the capabilities to assess the burst metadata and fast survey data across the constellation and to select the burst data buffers for downlink that have the highest probability of meeting the science goals of the mission. Once the desired burst data are selected, the SOC will generate the commands needed to configure each observatory in the constellation to preserve the selected data and set the priority of the data to be downlinked. These commands are uplinked to the observatory using a method called Store \& Forward $(\mathrm{S} \& \mathrm{~F})$, in which commands are delivered by the SOC to the MOC for deferred uplink on the next contact. In addition the SOC will collect the downlinked burst and science data, perform Level 1 processing, distribute the data to the science community, and archive the data.

- The SITL uses customized data viewing tools to evaluate onboard burst data and downlink priorities, making informed adjustments as needed.

\subsection{Nominal Burst Data Selection Flow}

The data selection process (see Fig. 6) involves several cooperating elements, each with an important role, as described below.

\subsubsection{Onboard Burst Data Management}

The CIDP Mass Memory Module provides 96 GB of data storage organized as 24576 buffers of $4 \mathrm{MB}$ each. These buffers are primarily used to store collected survey and burst data. Upon command, the CIDP telemeters Metadata containing the CDQ value for each buffer. After the ABS evaluates the CDQ data and selects sets of trigger cycles for downlink, commands are radiated to the Observatories to enqueue the corresponding data buffers for downlink. 


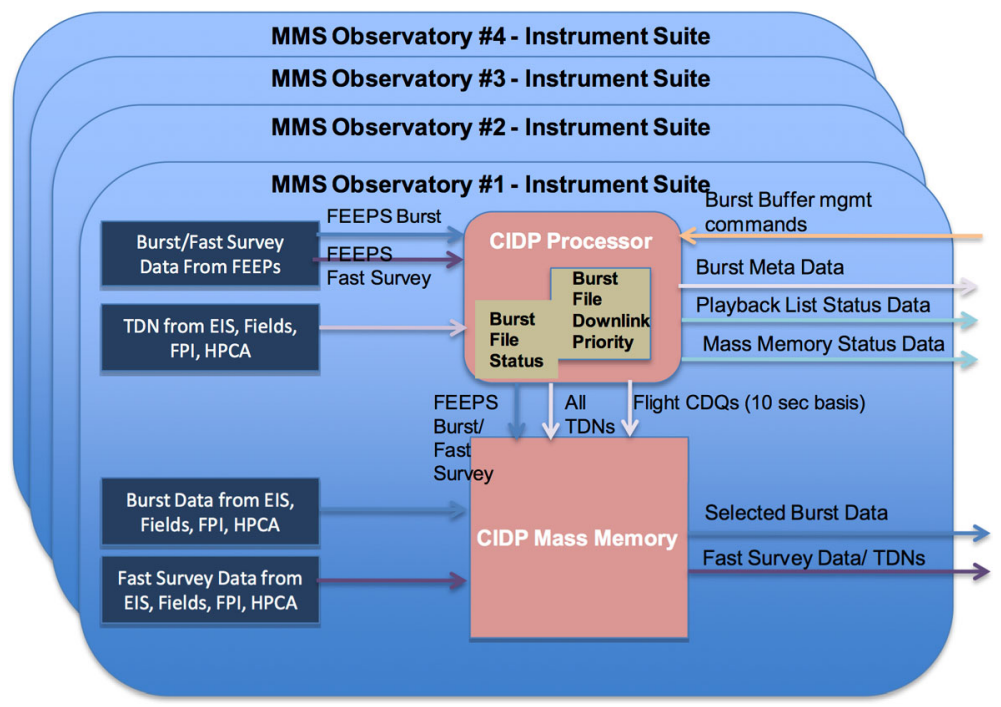

Fig. 6 Burst data collection and selection overview for the flight segment. Note that most instruments in the payload select survey and burst data that are to be presented to the central processor (CIDP), but the energetic particle package (FEEPS) is directly handled and processed by CIDP

Each Science Instrument generates Burst Data and sends it to the CIDP (Fig. 6). Simultaneously each also computes TDNs and sends them to the CIDP, as described previously. However, the CIDP computes Trigger Data Numbers for the FEEPS instrument from the raw data frames it receives. The CIDP then computes the Cycle Data Quality from the received and computed TDNs. The Burst Data received from each instrument is stored in a Burst File along with corresponding Burst Metadata, which includes the CDQ, the Trigger Cycle start time and Burst Buffer File Size (in bytes).

These data are held within the CIDP, which then manages the playback of Burst Files based on received ground commands.

\subsubsection{Ground-based Burst Data Management}

Figure 7 depicts in greater detail the processing blocks within the SOC that perform the Burst Data Management functions. The four main processing steps in Burst Data Management are Metadata Ingest, Downlink Status Processing, Data Segment Definition and Selection, and Command Generation.

The ground-based portion of the Automatic Burst System is the Burst Data Management (BDM) system. Using the results from the automatic selection algorithm or optional inputs from the SITL, BDM manages buffer allocations and downlink queues in each CIDP. The key management element is the Burst Data Segment and its associated FOM, which have been introduced earlier. This abstraction ties together sets of burst buffer files from all four observatories and allows prioritizing them according to their likelihood of containing interesting science data.

Several data segments containing burst data files that are held (protected from being overwritten) in the CIDP Mass Memory and may be selected for downlink. To ensure adequate data storage for burst and survey data from subsequent orbits, some data files containing lower priority data are not held and are instead left in an unprotected state (in-use in CIDP 


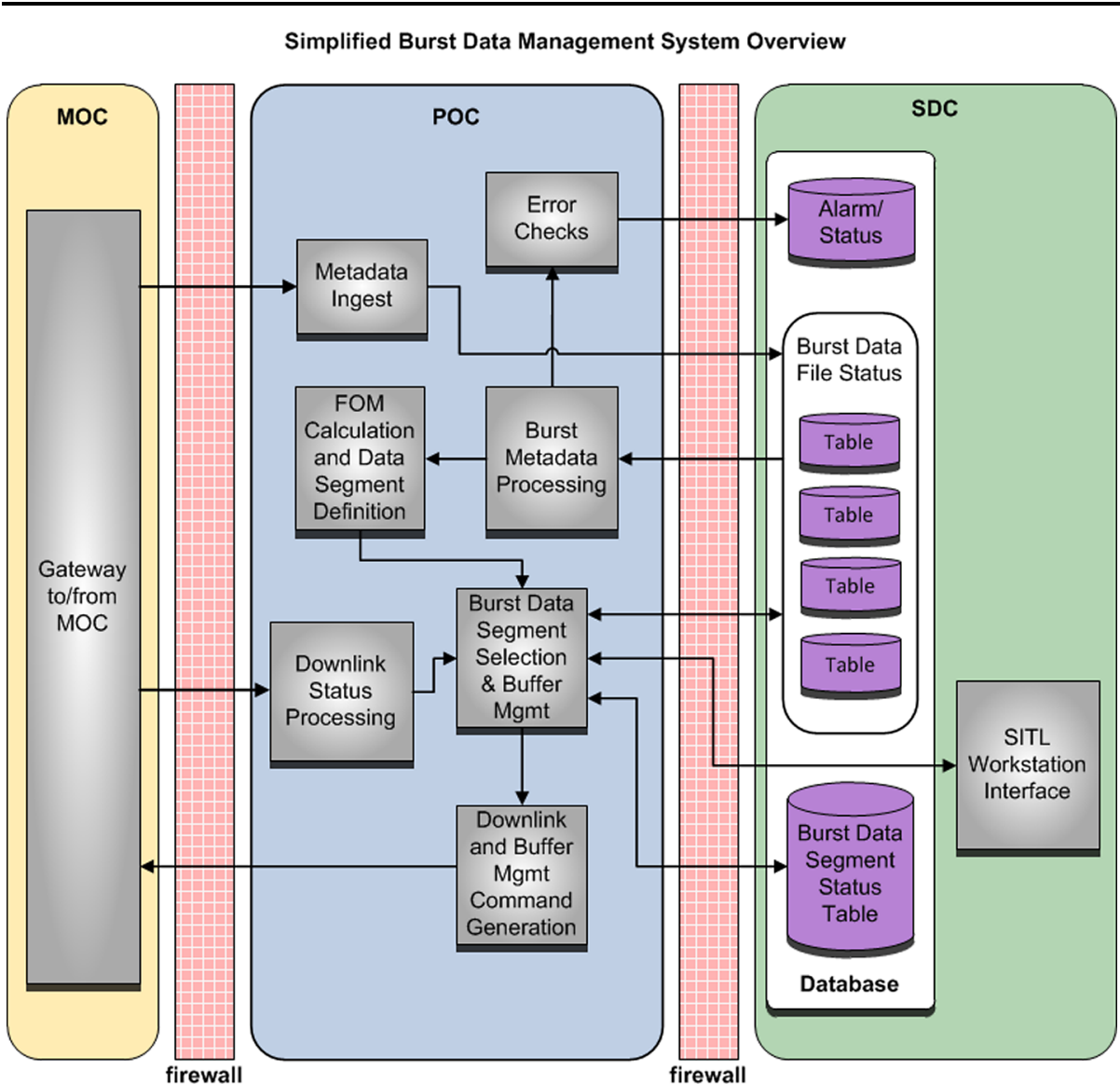

Fig. 7 The automated burst selection (ABS) data management system data flow from the Mission Operations Center through the Payload Operations Center to the Science Data Center

terminology) so they are available to be overwritten by new data. Data files may be from several different ROIs and can be stored in different areas within CIDP Mass Memory. The ground-based ABS maintains a mapping of data files on board each observatory to their defined data segments, as depicted in the Fig. 8.

\section{Data Access and Availability}

The MMS ground data system is designed to fully enable timely exploitation of the simultaneous measurements from all four MMS spacecraft, and there are no proprietary periods associated with any of the MMS-SMART data products. To help ensure that the most recent scientifically useful products (e.g. Level 2 and Level 3) are readily accessible, availability will be centralized at the SDC. The SDC will function as an active online data center, from which all MMS science products will be provided, as well as basic tools that aid in data access and analysis.

Given the significant MMS science data volumes, online data access interfaces will provide the ability to zero in on specific data of interest prior to download. For scientists work- 


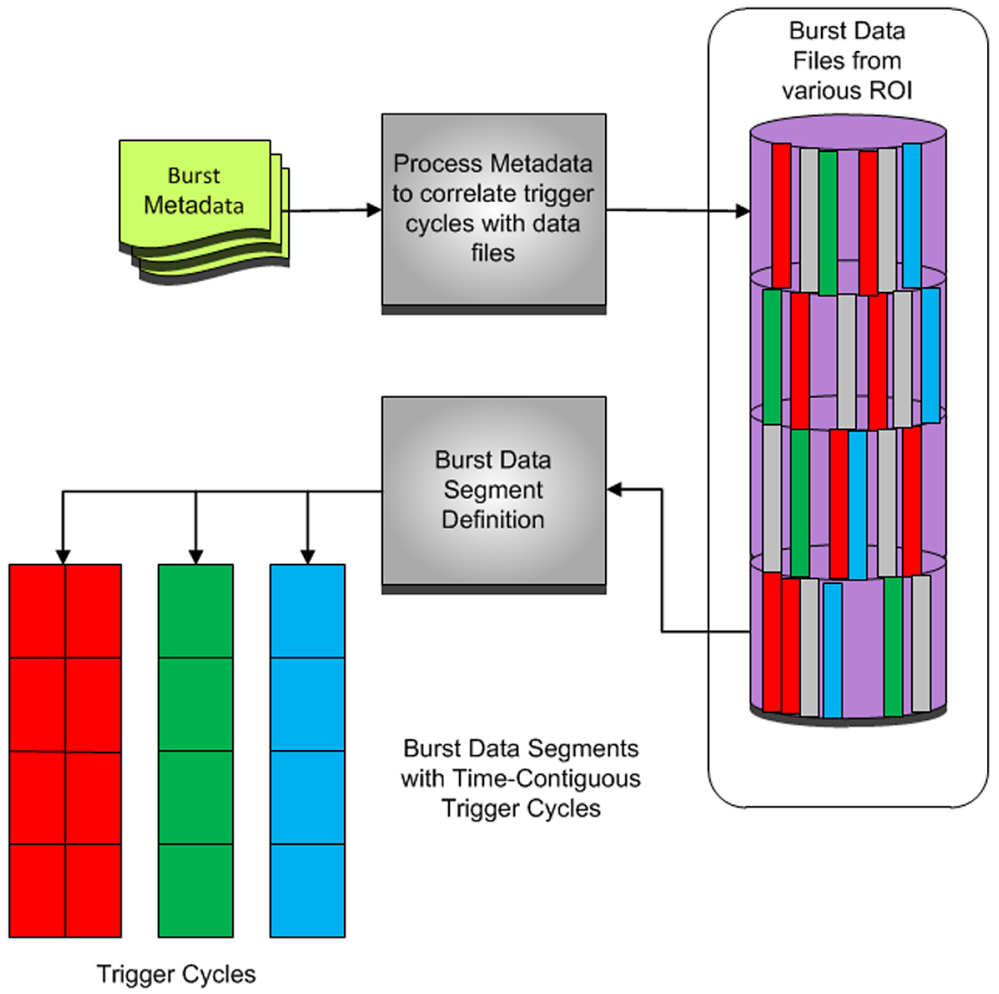

Fig. 8 Mapping burst data files to data segments. Burst metadata are used to relate trigger information with the relevant data files. Contiguous sets of 10 second trigger cycles and corresponding data files are logically combined into Burst Data Segments, which may be enqueued for downlink

ing with the data, this will minimize the amount of data that needs to be downloaded and managed on local systems.

The general science community will be able to access MMS science data products (Quicklook, Level 2, and Level 3) via a publicly accessible interactive web site, which will be maintained and hosted at the SDC. The SDC will also make data products available via RESTful "programmer level" or direct-access interfaces, which will enable access by "power users", who already know what they want, automated processing and analysis systems at other institutions, as well as Virtual Observatories (VxOs). In addition, MMS data are made available through existing SPDF multi-mission data services such as CDAWeb and orbit services such as SSCWeb to further enable multi-mission science studies using MMS data and to more easily use data from other missions to establish the larger context for given MMS observations.

In close coordination with the MMS science groups, tools and visualizations that are determined after launch to have significant merit and broad applicability will be made available from the SDC. In this way, MMS can capitalize on cross-cutting tools, such as those that are tailored for close association with modeling efforts or in other ways exploit the unique MMS data set.

General Public users will be able to access data at the SDC website but may be better served by the Education and Public Outreach (EPO) website hosted at Rice University (see Fig. 1). The EPO team at Rice plans to make high-level MMS science easily accessible to 
Table 9 MMS science data availability timeline. Routine availability is measured relative to receipt on the ground, usually 1-3 days post-measurement, depending upon mission phase

\begin{tabular}{llll}
\hline Data type & Access & Initial Availability & Routine Availability \\
\hline Level 1 & MMS Internal Interfaces & Commissioning & $\begin{array}{l}\text { Survey: 1-hour latency; } \\
\text { Burst: 24-hour latency } \\
\text { QuickLook }\end{array}$ \\
Level 2 & Public via SDC & No later than 6-months & 24-hour latency \\
Level 3 & Public via SDC & $\begin{array}{l}\text { Following the end of } \\
\text { commissioning }\end{array}$ & 30 day latency \\
\hline
\end{tabular}

journalists, teachers, and students through animations, data visualizations, workshops, and sonifications.

A timeline for MMS data availability is summarized in Table 9.

\subsection{Science Data}

The SDC makes available QuickLook, Level-2, and Level-3 science data to the science community and general public. MMS instrument teams provide the SDC with software to produce QuickLook data products and also Level-2 data files for archival and distribution.

In order to most effectively index, store, and distribute the MMS-SMART data, instrument teams also provide the SDC with documentation and metadata for each of their products. These science products are made available to the science community through a data portal accessible from the SDC web site. Documentation and metadata will support the Space Physics Archive Search and Exchange (SPASE) data model (Thieman et al. 2010) so that the SDC can also readily provide access to the data through Virtual Observatories (VxOs). The SDC collaborates with applicable VxOs to ensure that MMS data are also accessible through these channels.

Additionally, the SDC maintains a record of spacecraft ephemeris and attitude information, instrument operating parameters, other ancillary data, and version history notes for each data product and provides access to these data via its data access website.

To help the science community find data, the SDC will provide a web-based data availability browser. This interactive tool displays a "fever chart" of available data products and highlights potential issues to be investigated by data processing engineers. A notional sketch of the tool is depicted in Fig. 9.

\subsection{Engineering Data}

Spacecraft and instrument suite housekeeping/engineering data have minimal scientific value and may also be subject to export restrictions. Many of these data are only available to MMS team members and other designated individuals or organizations; however, effort is made to ensure that all housekeeping and engineering data that are necessary to support the use and interpretation of MMS science data are made publicly available from the SDC web site, along with the MMS science data products.

\subsection{Data Access Software}

In coordination with the MMS science team, the SDC will provide access to interfaces and software tools to allow basic display and manipulation of MMS science data. These 


\begin{tabular}{|c|c|c|c|c|c|c|c|c|c|c|c|c|}
\hline Burst & & Survey & \multicolumn{2}{|c|}{ Help } & & & & \multicolumn{4}{|c|}{ October $10 / 11->10 / 20$} & \\
\hline$\sum_{\Sigma}^{\infty}$ & $\begin{array}{r}\text { FPI } \\
\text { FIELDS } \\
\text { HPCA } \\
\text { EPD } \\
\text { ASPOC } \\
\text { Data Level }\end{array}$ & 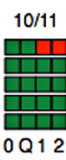 & 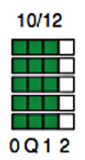 & 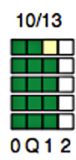 & 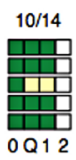 & 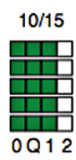 & 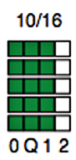 & 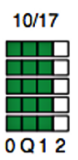 & 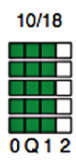 & 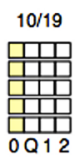 & ${ }_{10}^{10}$ & $\frac{20}{\frac{1}{1}}$ \\
\hline$\sum_{\sum}^{N}$ & $\begin{array}{r}\text { FPI } \\
\text { FIELDS } \\
\text { HPCA } \\
\text { EPD } \\
\text { ASPOC } \\
\text { Data Level }\end{array}$ & 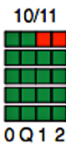 & 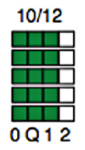 & 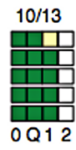 & 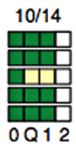 & 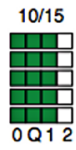 & 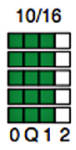 & 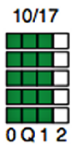 & 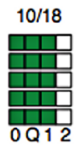 & 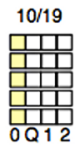 & 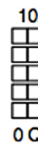 & \\
\hline$\sum_{\sum}^{\infty}$ & $\begin{array}{r}\text { FPI } \\
\text { FIELDS } \\
\text { HPCA } \\
\text { EPD } \\
\text { ASPOC } \\
\text { Data Level }\end{array}$ & 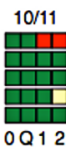 & 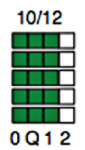 & 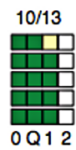 & 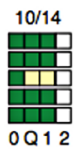 & 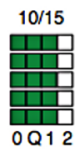 & 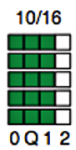 & 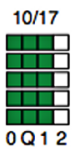 & 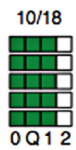 & 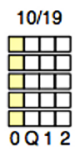 & $\begin{array}{l}10 \\
\text { प्र } \\
\square \square \\
\square \\
00\end{array}$ & \\
\hline$\sum_{\Sigma}^{D}$ & $\begin{array}{r}\text { FPI } \\
\text { FIELDS } \\
\text { HPCA } \\
\text { EPD } \\
\text { ASPOC } \\
\text { Data Level }\end{array}$ & 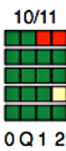 & 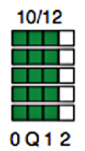 & 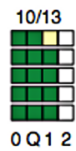 & 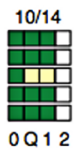 & 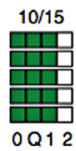 & 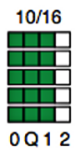 & 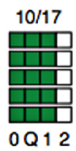 & 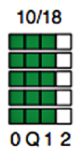 & 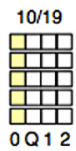 & $\begin{array}{l}10 \\
\square \square \\
\square \\
\square \\
00\end{array}$ & \\
\hline
\end{tabular}

Fig. 9 Representative data availability browser for the several instruments on the four MMS spacecraft. The intention through this "fever chart" format is to show day-by-day what data at each level are available to users

tools will be made available to the science community via the SDC's data access website concurrently with the initial public data release indicated in Table 9.

\section{MMS Instrument Suite Operations}

Over the course of an orbit, the complement of MMS instruments perform a highly orchestrated sequence of operational and observational activities to maximize the science data return and quality from the MMS observatories. As described in Fuselier et al. (2014, this issue), the MMS mission will encompass two distinct orbit phases for primary science collection: Phase 1, which consists of a $1.2 \times 12 \mathrm{R}_{\mathrm{E}}$ orbit to enable day-side measurements, and Phase 2 , which consists of a $1.2 \times 25 \mathrm{R}_{\mathrm{E}}$ orbit for night-side measurements. A pictorial view of a Phase 1 nominal MMS orbit is depicted in Fig. 10 and a Phase 2 orbit is depicted in Fig. 11. These figures illustrate the key regions of the MMS orbit, as well as the operational events that take place.

Each MMS orbit is separated predominantly according to science and non-science (e.g. calibration) priorities. Science data collection occurs while the MMS constellation is within defined Regions of Interest (ROIs), whereas the other portions of the orbit are used chiefly for calibration, health/safety, and maintenance activities. Two ROIs are defined which drive both the operational and observational characteristics of the MMS nominal operations scenario: the Control ROI and the Science ROI (Fuselier et al. 2014, this issue). The Control ROI is defined as the region of the orbit in which the MMS constellation's formation is actively maintained, and is of primary interest to the MMS flight dynamics team, which actively maintains inter-spacecraft separation and the constellation's tetrahedron quality (Fuselier et al. 2014, this issue). For Phase 1 orbits the Control ROI encompasses the section of the MMS orbit where the spacecraft are at a distance $>9 R_{E}$. For Phase 2 orbits, it is $>15 R_{E}$. 


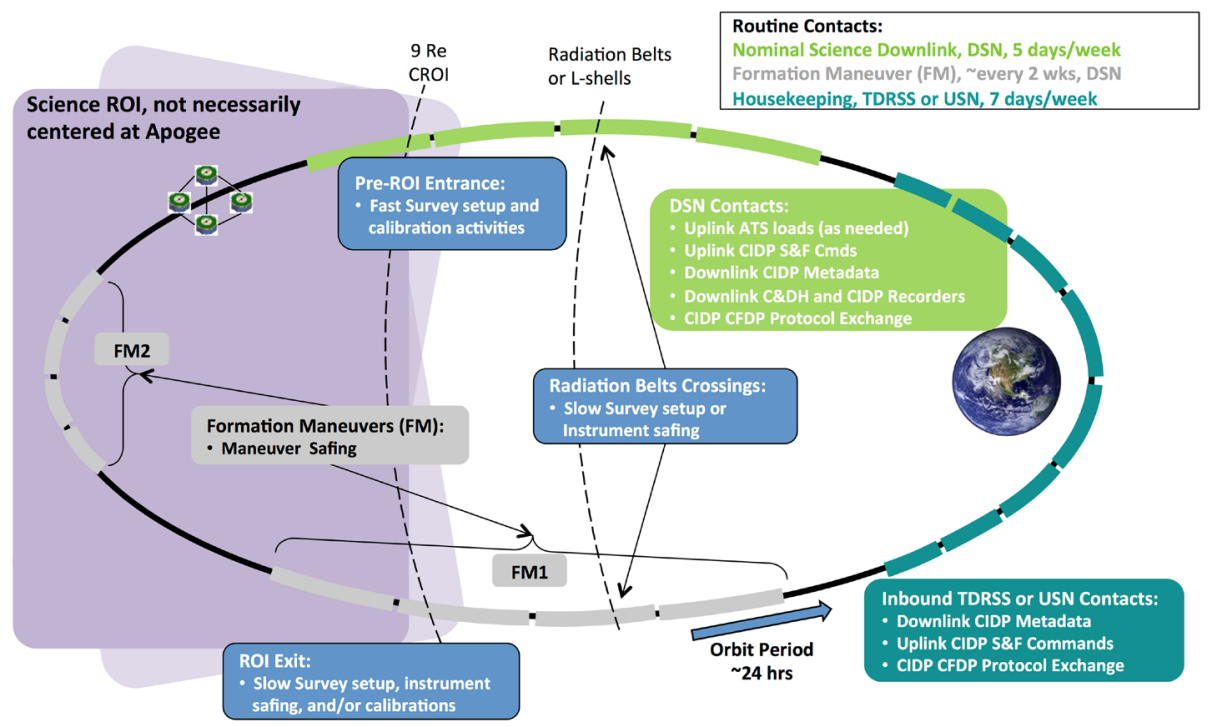

Fig. 10 MMS Phase 1 Orbit-in-the-Life. A Phase 1 orbit period is approximately 24 hours, over which the instruments perform primary science operations during the day-side Science Region of Interest and calibration/maintenance activities at other times. Formation Maintenance (FM) maneuvers are expected to occur periodically, but not every orbit, and several ground contact opportunities are planned each orbit

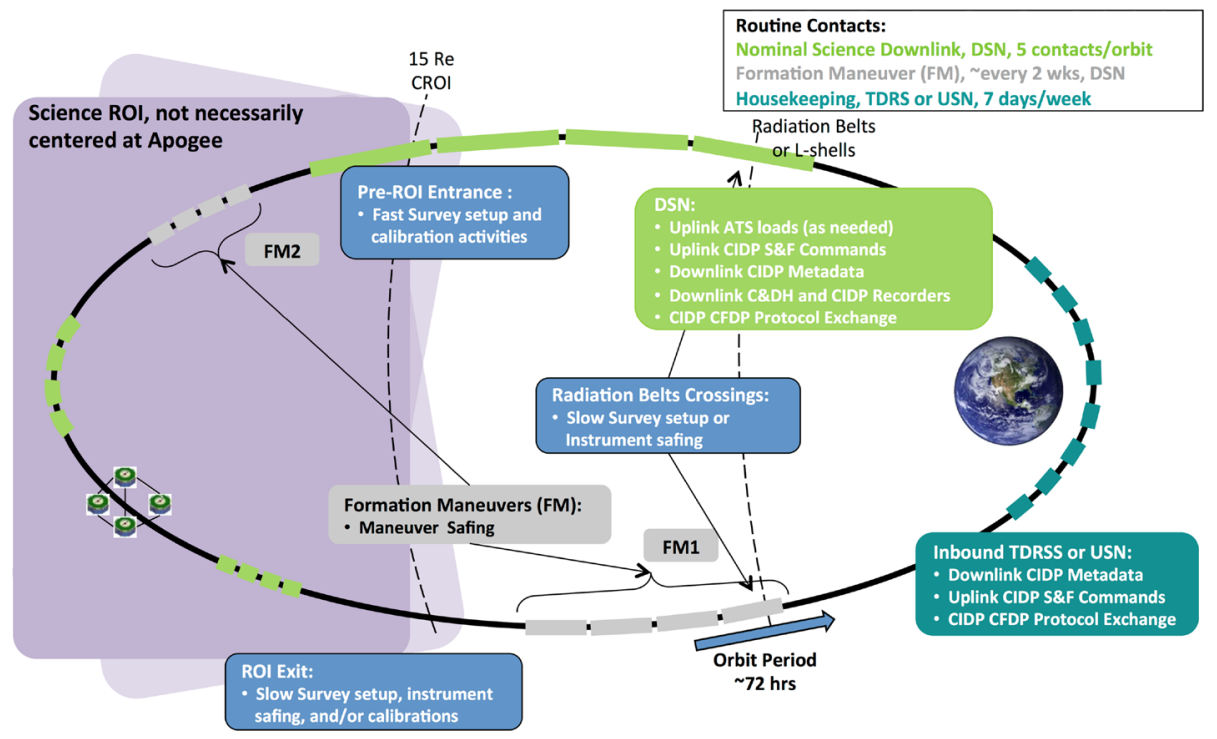

Fig. 11 MMS Phase 2 Orbit-in-the-Life. A Phase 2 orbit period is approximately 72 hours, over which the instruments perform primary science operations during the night-side Science Region of Interest. Calibration and maintenance activities are performed at other times, including occasional Formation Maintenance (FM) maneuvers. Numerous ground contact opportunities are planned each orbit 
In the Control ROI, the quality of the tetrahedron formation is maintained. The Science ROI is a subset of the Control ROI where the MMS instrument suites will be placed into their fast-survey data collection modes in order to collect primary science measurements. The Science ROI encompasses approximately $50 \%$ of the orbit period, and is allowed to shift from week-to-week according to expected magnetospheric conditions in order to optimize science data collection. Using a distinct Science ROI is also intended to optimize science collection relative to limited resources, such as expendables (e.g. ASPOC's indium supply) and onboard data storage capacity. The Science ROI definition is expected to be updated every 2-3 weeks, and is defined by start/stop event times, which serve as command triggers within the POC's planning and scheduling system.

In order for primary science data collection to occur within the Science ROI, the MMS instrument suites are configured into their primary Fast Survey science collection modes in advance of Science ROI entry. Instrument transition into Fast Survey mode is sequenced via the spacecraft's onboard Absolute Time Sequence (ATS) command load in a staggered fashion in order to maintain onboard command execution rate limits. To this end, science instruments are progressively commanded into their Fast Survey mode, with the CIDP (Tooley et al. 2014, this issue) commanded into this mode last, which then begins Fast Survey and burst data recording. Similarly, a staggered sequence executes upon Science ROI exit to place the instruments into their Slow Survey modes, thus ending the primary science collection for a given orbit.

In order to maximize science data quality and maintain the performance of the instruments, each instrument suite undergoes calibration activities during a nominal orbit. While the data collection that occurs during the Science ROI is relatively straightforward, the overall operations plan for MMS becomes more complex owing to the variety of mode transitions and associated coordination that is required at other times. In order to accommodate each instrument team's preferences for optimizing the timing, duration, and sequence of calibration activities, the logic for scheduling these activities is programmed into the POC planning and scheduling system. This system utilizes a highly capable logic engine, which provides significant flexibility in orchestrating complex arrays of activities in response to events or other triggers. In this way, the relatively complex sequence of instrument calibration and other operational activities, consisting of many hundreds of activities per orbit, is accommodated in an automated fashion.

In addition to nominal science and calibration activities, the MMS instruments suites must also respond to other important orbit events, notably spacecraft maneuvers and periods of eclipse, where the observatories will be within the Earth's shadow. Generally speaking, instrument suite maneuver operations are motivated to reduce any risk associated with operating high voltage systems while gaseous plumes from spacecraft thrusters are present. In advance of a planned maneuver, the CIDP is commanded via onboard ATS to issue a "maneuver safe" message to the instruments 30 minutes before the planned maneuver start. Instruments then autonomously respond and reduce their high voltage levels to predefined safe values. Following a maneuver activity, instruments are then commanded via ATS to their normal operating modes. The planning and scheduling system at the POC uses orbit event inputs received from the MOC and FDOA to generate the ATS command sequences necessary for maneuver entry and exit. This system is configured to handle all variations, including cases where the maneuver is planned to occur within or straddles the Science ROI.

Eclipse operations are another key operational situation that must be handled. The expectedly limited power resources available onboard the MMS spacecraft require that overall 
power draw from the MMS instrument suites be managed to levels that can be sustained during eclipses. To reduce complexity, a manageable three eclipse scenarios are defined, each of which has a defined operations response. These three scenarios are eclipses less than 2 hours in duration, eclipses 2-3 hours in duration, and eclipses greater than 3 hours in duration. Selected instruments and/or their heaters are powered down or completely off according to predetermined plans if the eclipse duration is less than 3 hours. For eclipses longer than 3 hours, the entire instrument suite is powered off to conserve power.

\section{Data Archive}

The SOC serves as the Mission Archive during the MMS mission, having responsibility for archiving all MMS mission data, and will help plan and provide for subsequent archive phases prior to eventual mission termination. Backup and recovery mechanisms are employed at the SOC and include contingency plans for ensuring a full recovery from catastrophic damage or failure. This includes offline and offsite backup of all irreplaceable information, including software, calibration data, and other system dependencies. Resources archived at the SOC include:

- Data-raw instrument and spacecraft telemetry (housekeeping and science); calibration data; ephemerides; attitude and other ancillary data needed to support scientific use and interpretation of MMS data; all levels of science data products.

- Burst system metadata including burst quality values (e.g. TDNs, CDQs, MDQs, and FOMs); Automated Burst System selections; SITL selections. Even though these data will not be useful for selecting onboard data once it has been overwritten, this data could be very useful for defining future data selection algorithms.

- Metadata and Documentation describing data products (e.g. in SPASE terms), algorithms, instrument calibrations, validation, and data quality; also, command and telemetry definition information, descriptions of spacecraft and instrument design and operations, status reports, and other information needed for use of MMS data.

- Software and analysis tools, including software used for generation of scientific data products and corresponding metadata, as well as tools used for accessing, visualizing, and interpreting MMS science data.

The SOC collects and archives MMS data beginning with the pre-launch testing phase, principally telemetry data generated during ground testing and calibration activities. Following launch, as instruments are commissioned and begin routine science data collection, the SOC is actively responsible for data production and distribution activities and the archiving function associated with management of science data sets. Throughout the mission, the SOC ensures that adequate security and backup/recovery mechanisms are established and documented, thereby maintaining the integrity of all data managed in the SOC repository and safeguarding them against loss. As responsibility for data processing and analysis activities is a distributed function on the MMS mission, each MMS-SMART instrument team also shares responsibility for maintaining the integrity of the MMS data during the active mission. The SOC and all ITFs will use backup/recovery mechanisms and will maintain contingency plans for achieving a full recovery from catastrophic damage or failure.

At the SOC, multiple mechanisms are used to ensure data retention and integrity. The POC utilizes a tape-based backup system, along with a third-party offsite archiving approach 
for all operations and low-level telemetry data. The MMS SDC, also part of the SOC, uses a Cloud-based solution called Amazon Glacier for offline, offsite, redundant storage of MMS science data products and associated information. By the end of the prime MMS mission, roughly 100 terabytes (TB) of science data will be managed. Comparative evaluation of local and managed Cloud-based solutions was performed, with the Cloud-based solutions available at the present time providing both greater flexibility and overall cost effectiveness than more traditional approaches.

\section{Data Rights and Rules for Data Use}

The terms for distribution and use of the MMS data products are specified in the NASA Heliophysics Science Data Management Policy document (Fisher 2009), and are summarized here in terms of the MMS mission. These guidelines govern both the production and distribution of scientific data sets by the MMS program, and also use of the MMS data by the science community and general public, and are summarized below:

- MMS-SMART scientifically useful data products are open to all scientists and the public (Users).

- There are no proprietary periods associated with any of the MMS-SMART data products.

- Users shall have timely access to the scientifically useful data and analysis tools that are equivalent to the level that the MMS-SMART science team uses.

- All potential data users are strongly encouraged to contact the MMS-SMART Principal Investigator (PI) or a designated lead investigator of an instrument or modeling group early in an analysis project to discuss the appropriate use of instrument data or model results.

- All users who wish to publish results derived from MMS data are strongly encouraged to directly involve the MMS-SMART PI and/or Instrument Leads and team members as appropriate in the analysis and to offer co-authorship. Data users who are members of the MMS-SMART science team are required to offer co-authorship. Co-authorship may be declined.

- Users should heed the caveats of investigators as to the interpretation and limitations of data or model results. All important caveats should be included in the publication, even if co-authorship is declined. Data and model version numbers should also be specified.

- Users should acknowledge the sources of data used in all publications, presentations, and reports. Appropriate acknowledgement to institutions, personnel, and funding agencies should be given.

- Users are encouraged to provide the MMS-SMART PI and/or instrument PIs a copy of each manuscript that uses MMS-SMART data upon submission of that manuscript for consideration of publication. On publication the citation should be transmitted to the PI and any other providers of data.

- MMS encourages that pre-prints of publications and conference abstracts be widely distributed to interested parties within the mission and related projects.

- Users are encouraged to make tools of general utility and/or value-added data products widely available to the community. Users are encouraged to notify the MMS-SMART PI of such utilities or products. The User should also clearly label the product as being different from the original MMS-SMART produced data product. 


\section{Conclusion}

The MMS mission features burst data that have cadences that can resolve the electron diffusion region in magnetic reconnection. The burst data volume, however, far exceeds the bandwidth of the MMS telemetry transmission system. The MMS mission is designed to store on board several orbits of data in the science regions or interest. A small fraction of the stored data with the highest scientific quality, that is, the highest likelihood of being associated with magnetic reconnection is selected for transmission. The data selection process is automated using on-board trigger data supplied by the instruments, which indicate a higher probability of reconnection events. In addition, a manual SITL selection process allows scientists to use other data not available to the automated process to select time periods of the high-cadence burst data that are likely to be associated with magnetic reconnection.

MMS data will be generated and disseminated using a multi-institution operations and data system, which distributes overall responsibility among the various MMS team institutions. A mission of this scale and complexity demands a coordinated team approach, which can simultaneously provide uniformity to the end-users in the science community. The MMS ground system is designed to balance these elements of a complex and groundbreaking mission in order to provide the highest quality and comprehensive in-situ scientific measurements of the Earth's magnetosphere to-date.

Acknowledgements The authors thank Southwest Research Institute (SwRI) leadership and the many science investigators on the Magnetospheric Multiscale mission for their cooperation. Thanks also go to management and operations colleagues at NASA Goddard Space Flight Center (GSFC). We especially acknowledge the efforts of mission operations and data systems personnel at the Laboratory for Atmospheric and Space Physics. This work was supported by SwRI subcontract 599791Q under NASA program NNG04BB99C (Project \# 1541955).

Open Access This article is distributed under the terms of the Creative Commons Attribution License which permits any use, distribution, and reproduction in any medium, provided the original author(s) and the source are credited.

\section{Appendix: Terminology and Definitions}

The following terminology is used to describe the MMS Burst System and aids in understanding the detailed discussion in Sect. 4.

Burst Buffer: The physical storage of the Burst File in the CIDP Mass Memory.

Burst Data Collection Region: The area within the ROI where fast survey and burst data are collected. This will nominally be one-half the orbit period during each phase, $\sim 12$ hours for Phase 1 and $\sim 34$ hours for Phase $2 b$, and will occur within the boundaries of the ROI.

Burst Data Segment: A collection of ' $\mathrm{N}$ ' time-contiguous 10-second burst files. The value ' $\mathrm{N}$ ' is determined in the Figure of Merit calculation. The Burst Data Segment contains burst files from all observatories across the same time range, thus providing a uniform data set containing data from all instruments across all four observatories.

Burst File: Burst data for one Trigger Cycle (10 seconds) collected from the entire instrument suite on one observatory (4 MB).

Burst Metadata: Start time of recording, cycle data quality, and size for each Burst File. Burst Metadata can be downlinked in real-time telemetry, but is also stored on the spacecraft recorder for downlink at a subsequent ground contact. 
$C D Q$ (Cycle Data Quality): An indicator (number in the range 0 to 255) of the quality of the data in a Burst File, which is, computed every Trigger Cycle from one or more trigger data numbers. There are four CDQ values every 10 seconds, one for each spacecraft. It is planned for this quantity to be calculated on board by the central processor. This item can also be calculated on the ground by the SOC or by a scientist by using the downlinked information.

Constellation: All four Observatories.

Designated Scientist: Scientist responsible for verifying and updating Burst Data Segment definition and prioritization on behalf of the science team, a role that is carried out manually daily during Phase 1 and several times a week in Phase $2 b$.

Fast Survey Data: Survey data collected simultaneously with burst data, during the period of the orbit containing the ROI. It may be used by scientists to further refine selection of Burst Data Segments.

Figure Of Merit: An assessment of the quality of the Burst Data Segment in terms of the likelihood that it contains a magnetic reconnection event. The FOM is a single value, from 0 to 255, for a Burst Data Segment representing the constellation. The FOM calculation algorithm will be executed at the SOC.

Instrument Suite: All science instruments on any one observatory.

Magnetic Reconnection Event: See Burch et al. (2014, this issue) for a full definition.

Mission Data Quality: A quantity in the range 0 to 255 that is derived from a sort-weighting of the four CDQ values and are assigned to each 10 second burst interval. The MDQ is a single value representing all four spacecraft (the constellation). This quantity will be calculated on the ground by the SOC.

Observatory: The combination of one MMS spacecraft and its instrument suite.

ROI (Region Of Interest): The regions of science interest are defined as geocentric apogee distances $>9 R_{E}$ when in the magnetopause for Phase 1 , and $>15 R_{E}$ when in the nightside neutral sheet for Phase 2.

Scientist in the Loop (SITL): A capability implemented in ground software and carried out manually by the designated scientist, whereby onboard burst data can be evaluated and downlink priorities can be adjusted.

Trigger Data Number: a number in the range 0 to 255 that may be used as an indicator of the quality of the burst data acquired by an individual instrument. The value 0 indicates the lowest quality and the value 255 indicates the highest quality. Several TDNs are computed by each instrument and provided to the CIDP each trigger cycle. Since FEEPS does not include a processor, the CIDP calculates TDNs for this instrument.

Trigger Cycle: a ten-second segment of time, which is synchronized across the MMS constellation to within the uncertainty of the individual time reference $(\sim 1 \mathrm{~ms})$ on each observatory.

Trigger Data: Scientific measurements used to compute TDNs each Trigger Cycle.

\section{References}

M.H. Acuña et al., The global geospace science program and its investigations. Space Sci. Rev. 71, 5-21 (1995)

J.L. Burch, T.E. Moore, R.B. Torbert, B. Giles, MMS overview and science objectives. Space Sci. Rev. (2014, this issue)

R.E. Ergun et al., The FAST satellite electric field and magnetic field instrument. Space Sci. Rev. 98, 67-91 (2001)

R.R. Fisher, NASA Heliophysics Science Data Management Policy: http://hpde.gsfc.nasa.gov/Heliophysics_ Data_Policy_2009Apr12.pdf 
S.A. Fuselier, W.S. Lewis, C. Schiff, R. Ergun, J.L. Burch, S.M. Petrinec, K.J. Trattner, Magnetospheric multiscale science mission profile and operations. Space Sci. Rev. (2014, this issue). doi:10.1007/ s11214-014-0087-x

B.H. Mauk et al., The Energetic Particle Detector (EPD) investigation and the Energetic Ion Spectrometer (EIS) for the Magnetospheric Multiscale (MMS) mission. Space Sci. Rev. (2014, this issue). doi:10.1007/s11214-014-0055-5

C.J. Pollock et al., FPI instrument paper. Space Sci. Rev. (2014, this issue)

J.R. Thieman et al., SPASE and the heliophysics virtual observatories. Data Sci. J. 9 (2010)

C. Tooley et al., MMS observatory. Space Sci. Rev. (2014, this issue)

R.B. Torbert et al., FIELDS instrumentation suite on MMS: scientific objectives, measurements, and data products. Space Sci. Rev. (2014, this issue)

K. Torkar et al., Active spacecraft potential control investigation. Space Sci. Rev. (2014, this issue). doi:10.1007/s11214-014-0049-3

D.T. Young et al., Hot plasma composition analyzer for the magnetospheric multiscale mission. Space Sci. Rev. (2014, this issue). doi:10.1007/s11214-014-0119-6 